\title{
Critique of Research Methods in Chinese Translation Studies
}

\author{
Hongxiu Liu \\ School of Foreign Languages, Wuhan Polytechnic University; PhD. Student of UWTSD \\ Email: liuhongxiu0318@163.com

\begin{abstract}
This article first reviews the historical background, strengths and limitations of both qualitative research methods and quantitative research methods in applied linguistics, especially Chinese translation studies. Then the article proposes the mixed methods research as a better way to do future Chinese translation studies with suggested case studies.
\end{abstract}

Keywords: critique, qualitative research methods, quantitative research methods, translation studies

\section{INTRODUCTION}

In this article, qualitative research approaches and quantitative research approaches will be discussed from their respective historical background to their advantages and disadvantages with special reference to translation studies. Then mixed methods will be introduced later and a few case studies of mixed methods research are proposed in the end to scaffold how to integrate qualitative and quantitative methods, especially in Chinese translation studies.

\section{CRITIQUE OF QUALITATIVE AND QUANTITATIVE RESEARCH}

The history of qualitative research could be sourced back almost a century ago in social science, It was mainly used to explain and support quantitative studies after 1930s and especially post-WWII period. "It was Glaser and Strauss who first defined qualitative methodology in the late 1960s" [5], which began to set a dominant place of qualitative research and posted as a challenge to the quantitative hegemony. Especially since the mid-1990s, the proliferation of qualitative research emerged and lent itself to growing acceptance in applied linguistics.

\subsection{The features of qualitative research}

According to the definition given by John Crewell[4], we could infer the following characteristics:

\subsubsection{Individuality-concerned}

For research subjects, it aims at addressing a particular social problem for a particular group of human beings or certain kinds of individuals, mainly concerning their feelings, psychology or social behaviors. Thus some scholars commend qualitative research as contextual, humanistic and ethical-friendly.

\subsubsection{Interpretative/exploratory}

In terms of research purpose, it unearths the detailed meaning of the data, explores how the phenomenon comes into being and explains why it occurs. It mainly relies on the researchers' interpretation and explanation of the data collected. Thus it's more descriptive and exploratory.

\subsubsection{Inductive}

In terms of research process, it inducts theories or assumptions from the data analysis of those participants, rather than setting up a hypothesis at the beginning of the research. Moreover, qualitative research could be penetrated with the following pros and cons.

\subsubsection{Flexibility Vs. less validity}

For one thing, qualitative research exhibits flexibility in setting as social phenomena occur naturally, and researchers are not manipulative in the context under study. Besides researchers are more likely to be responsive to the emergent occurrence of particular variations from participants or changes in their conducted 
study, especially study involving fieldwork, and those changes during their study might divert the focus of the research from its original, For another, a flexible written structure is also found in their final report as mentioned above. Thus many scholars regard it as a process of discovering, refining and developing theories. More subjective to some social threats, like the emergence of participants and other changes, or it may lead the research into an unknown journey and yield no productive predictions; It is also highly susceptible to researchers' educational background and biases, thus sometimes its validity might be diluted.

\subsubsection{Uniqueness Vs. less generalizability}

Qualitative research studies the micro understanding of social phenomena, sometimes specific case studies or small groups of samples are selected. It boasts in its specialty, and yet also means it has a smaller audience, thus more difficult to produce general effects.

\subsubsection{Complexity}

Complexity refers to qualitative research adepts at dipping into more complex situations and shedding light on complex human perceptions, feelings and thoughts on the one hand, and it also takes more time and energy to research on the other hand. Bearing the pluses and minuses of qualitative research in mind, many scholars attempted to find remedies, such as quantitative research, which might neutralize some flaws.

\subsection{The features of quantitative research}

Quantitative research could be dated back almost several centuries ago in social science. First inspired by the advancement of natural sciences in the $19^{\text {th }}$ century, It was not until "at the turn of the $20^{\text {th }}$ century, Francis Galton who underpinned quantitative data collection and analytical methods in psychology" [5]. The middle of the last century witnessed the dominance of quantitative research in social science, which was later challenged by qualitative research in the 1970s. It was still on the way of increasing sophistication later on in applied linguistics. The features could be summarized by John Crewell's definition[4] as follows:

\subsubsection{Number-centered:}

In terms of research subjects, it tends to include a wider scope of participants and build up a large database, then collect the data, analyze the data and use the data to test its original assumption. Thus, some scholars claim numerical feature as the most prominent and indispensable in quantitative research.

\subsubsection{Confirmatory:}

For research purpose, it usually puts forward a hypothesis at the beginning, then undergoes a series of experiments to test the hypothesis. It confirms whether the hypothesis is true or false from different aspects. It also states how many variables should be chosen and how much the relationship among them.

\subsubsection{Deductive}

For research process, it tries out experiments or pilot studies to test the correlation between variables, and then the result from the large database collected can draw some kind of conclusion. Besides, its following strengths and weaknesses could be scrutinized.

\subsubsection{Replicability Vs. less in-depth}

As the most is conducted in artificial settings, similar experiments could be replicated by other researchers given the variables controlled. Hence, the utilization of the research outcome could be generalized to more audiences. Meanwhile, due to a large number of samples, it mostly just touches the macro features of those samples, and sometimes in-depth micro detailed observation is ignored.

\subsubsection{Validity Vs. too much rigidity}

As its process is more data-based, researchers are more liable to be objective, independent from their personal biases and experiences. With a large database, the research results are more credible and valid compared with results from only a few case studies and researchers' involvement. With a distant and cold eye to the database and the process, the written report is all set up in a certain structure and there left not much room for researchers' autonomy. The rigid structure of quantitative research may lead to the insensitivity of causes of phenomena or some contextual changes.

\subsubsection{Simplicity}

Compared with qualitative individual studies, quantitative research may choose large computerized data networks or tools to conduct experiments, thus it's easier, quicker, more practical and time-saving. It's also accompanied by its inadequacies. Some scholars argue that it might be too simple, too general and too superficial because it averages out and sacrifices the variety of individual participants and their uniqueness.

\subsection{The qualitative and quantitative research in Chinese translation studies}

Traditional Chinese translation studies to a great extent were modeled on the qualitative method in the last 
century [11]; Qualitative research involves theoretical and applied studies, such as translation teaching, textual analysis and transcription. The $21^{\text {st }}$ century has still witnessed substantial qualitative research by cognitive and cultural approaches, descriptive investigations and bibliometrics. Among all the research funded by The National Social Science Fund of China (later NSSF in short), "qualitative research still dominates Chinese translation studies, and will still play its big role as the cultural turn in translation took place in recent years", [12], but the total amount is descending in recent five years [10].

The declining number of qualitative research is somehow caused by the interaction between methodology and computer technology, cognitive science, neuroscience and translation process. More empirical translation studies were born after the 1990s. The booming quantitative methodology in translation studies is realized mainly through process orientation, such as “corpus analysis, eye tracking, keystroke logging [6]". Glimpsing the latest five-year trend of research sponsored by NSSF, "the number of quantitative research papers in translation is increasingly rapidly" [10], though still not equivalent to its counterpart. Data is gathered primarily textually, experimentally and multimodeled [14].

The number of quantitative research in translation grows also because it shines in the following aspects, for instance, translation studies grounded on a large database might fuel their systematicity and objectivity, or empirical studies through quantitative method might disclose the rule in translators' choice of words, syntactical and textual features and scientifically refine the translation strategies. But some conventional scholars still hold the view that there are substantial data in translation that can't be measured numerically, such as the awareness of poetics, cultivation of sponsors and translators, translators' attitude and values [14], etc. Moreover, quantitative research would sacrifice the rich humanistic connotation of translation and just fall into the flow of natural science without a second thought. Without variables properly controlled, it would even deviate further from its original assumption. Disputes about the dichotomy of two kinds of research methodologies gave rise to mixed methodology.

\section{PROPOSED MIXED METHODS}

\subsection{The justification of mixed methods}

First, for research outcome, mixed methods research with proper guidance could merge the edges of two methods, offset their respective limitations and take advantage of both merits As some researchers indicate, "it is not enough to fulfill the purposes of a study with just fact and meaning, QUAN and QUAL, or numbers and language or even those combined, as quantitative research searches for numeric facts, whereas qualitative for language-based meaning" [9]. Research problems are better answered in terms of length and width. It may provide better insight to "multi-layers of meaning" and "different dimensions of facts" [8].

Second, in terms of research methodology disagreement, mixed methods research eases the tension between two hostile methodological battlefields, reduces the conflicts and buffers them against the attacks and slanders from each other. By narrowing down the gap and divisions between the two methods, it harmonizes the academic atmosphere and stands a better chance of setting up an amiable interdisciplinary or crossdisciplinary research trend. According to "Bryman's study on 232 social science articles, it reveals that multistrategy research is more commonly practiced" [3].

Third, from the researchers' side, mixed methods research renders researchers' lifelong training and learning opportunities and slows down their obstinacy and presumption. Whether self-access or systematical methodological training to skill improvement, mixed methods research will endow researchers with the latest updates in academics, confer them with more competence, and finally researchers may gain an upper hand in their research performance. In all probability, they are less opinionated to hold their own thoughts all the time and refuse to accept questions from opponents.

Fourth, for academic influence and dissemination, compared with either one method, it goes without doubt that mixed methods research could exert more impact on readers or followers, spread ideas far wider to people who might translate the research results into practice.

Finally, for one thing, it's better that research papers or books get accepted, peer-reviewed and published by more publishers within a shorter period of time. Comparatively speaking, mixed methods research opens more doors of publishing, with both two different kinds of journals into its publishing basket. For another, research is better done with financial support especially funds from institutions or government, therefore researchers won't be bothered with financial concerns on the way of conducting their studies, and financial support could slim the odds of financial insufficiency at the expense of research efficiency. Mixed methods research helps researchers earn more chances of financial endorsement from different kinds of organizations, as it states in Julia Brannen's paper---- “opportunities for mixed methods research are increasing with the rise in cross-national research in the context of the growth in European Union funding." [1]. The same case is also true of Chinese translation studies. It is said that "mixed methods research is in greater demand by NSSF" [10].

Since "translation has always been seen as a complex human activity and caused by a set of incentives" [11], a product of the cause-effect chain, many scholars suggest 
mixed-methods might be a new initiative to innovate Chinese translation studies.

\subsection{Proposed research designs of mixed methods and implications}

To maximize the mixed methods' beauty and minimize its defect, three basic models on how to mix quantitative and qualitative methods are depicted by John Creswell [4].

\subsection{1. "Convergent parallel mixed methods"}

To analyze the research issue comprehensively, the researcher collects and converges qualitative and quantitative data almost synchronically and then gives an overall interpretation of the whole results. For example, Zhu [13] analyzed six different Chinese versions of "The Adventures of Huckleberry Finn" from a lexical, syntactic and discursive angle. He presented the quantitative results with qualitative discovery side by side. It confirmed statistics with an inductive conclusion.

\subsection{2. "Explanatory sequential mixed methods"}

This kind of research starts with the quantitative method, based on which qualitative research is developed later to explain the previous results in detail. For instance, starting with corpus-based quantitative research on Dialogues in Hong Lou Meng, Liao [7] discovered the differences in words and syntax between Chinese and English versions, then a detailed qualitative study on four types of samples with remarkable differences is followed to unfold the possible causes. Thus the research problem is well explained with corpus data and causes further deepen the understanding of the problem.

\subsection{3. "Exploratory sequential mixed methods"}

Opposite to the second model, it initiates with a qualitative method and discusses participants' viewpoints and then analyzes the data and selects some appropriate information, such as some variables for later quantitative samples to do follow-up research. It is exemplified by Brazil's dissertation [2]. To get sound data on problems and its possible solution of C-E translation, interviews with renowned translators and translation professors in seven different Chinese universities were conducted first. Based on their views, the researcher designed some relevant questions in a follow-up survey for over 300 graduate students from different universities.

\section{CONCLUSION}

Irrespective of their strengths and limitations, qualitative, quantitative and mixed methods all contribute a lot to the prosperity of research in certain fields. The choice of research methodology largely depends on the specific research problem, researchers' personal preferences, experiences and their target audiences. Whatever method is chosen, what matters most is how it is utilized to serve its specific research purpose.

\section{AUTHORS' CONTRIBUTIONS}

This study is solely done and written by Hongxiu Liu.

\section{ACKNOWLEDGMENTS}

This study is under the sponsorship of Hunan Provincial Fund for Social Sciences (20WLH20).

\section{REFERENCES}

[1] Brannen, J.,(2005). Mixed Methods Research: A discussion paper. NCRM Methods Review Papers, NCRM/005

[2] Brazill, Sh.,(2016). "Chinese to English translation: identifying problems and providing solutions", Graduate Theses \& Non-Theses. 71. Master thesis, Montana: Montana Tech of the University of Montana,http://digitalcommons.mtech.edu/grad_rsc h/71 (assessed on Dec.12th, 2020)

[3] Bryman, A.,(2006). Integrating quantitative and qualitative research: how is it done? Qualitative Research. SAGE Publications (London, Thousand Oaks, CA and New Delhi). Vol. 6(1) 97-113.

[4] Creswell, J.,(2014). Research Design: Qualitative, Quantitative and Mixed Methods Approaches(4th edition). Thousand Oaks, CA: Sage.

[5] Dornyei, Z.,(2007). Research Methods in Applied Linguistics: Quantitative, Qualitative and Mixed Methodologies. Oxford: Oxford University Press

[6] Hu, Kb., and Han, Y.,(2017). The research status quo, problems and trend of translation in recent 15 years. Foreign Language Teaching and Research, Vol.49, No.2

[7] Liao, Ch.,(2019). A Quantitative and Qualitative Study of English Translation of Dialogues in Hong Lou Meng. PhD thesis. Shanghai: Shanghai International Studies University

[8] Litosseliti, L.,(2010). Research Methods in Linguistics. New York: Continuum International Publishing Group

[9] McLafferty, C., Slate, J., and Onwuegbuzie, A.(2010). Transcending the QuantitativeQualitative Divide With Mixed Methods. Counseling and Values, Oct 2010; 55, 1; ProQuest Central pg. 46-62

[10] Wang, Y., and Fan Wq.,(2020). Translation studies in the latest five years: A Statistical analysis of 
research projects funded by the National Social Science Foundation(2015-2019). Shanghai Journal of Translators, No. 5, P18-23

[11] Xie, K., and Zhou, N.,(2020). Qualitative Comparative Analysis: A New Approach to Translation Studies. Journal of Chongqing Three Gorges University, Vol.36, No.186

[12] Zhang, W.,(2015). Overview of translation studies in China: A Statistical analysis of research projects funded by the National Social Science Foundation(2000-2013). Foreign Language Teaching and Research, Vol. 47, No. 1(P.106)

[13] Zhu, Q.,(2013). A Methodological Proposal for the Evaluation of Translated Versions: A Case Study, The Adventures of Huckleberry Finn into Chinese. Overseas English, 2013(10)

[14] Zou, Cq.,(2020). Digital Humanity Research of Translation Studies from the Perspective of Big Data. Journal of Southwest Jiaotong University(Social Sciences), Vol.21, No.1 